\title{
Originals
}

\section{Role of CT in the evaluation of children with foregut cyst}

\author{
R.J.Hernandez \\ Department of Radiology, Section of Pediatric Radiology, University of Michigan Hospitals, C.S. Mott Children's Hospital, \\ Ann Arbor, Michigan, USA
}

\begin{abstract}
The presentation of a foregut cyst may vary from an asymptomatic mass discovered as an incidental finding in a chest radiograph, to signs or symptoms secondary to airway compression by the cyst. The radiographic evaluation of a child with a possible foregut cyst usually consists of a chest radiograph and barium esophagogram. Although this approach is often sufficient, in other instances delay of treatment occurs or unnecessary workups are performed due to the inadequacy of the approach. CT is useful for (a) depicting cryptic foregut cysts, (b) clarifying abnormal radiographs, (c) avoiding unnecessary workup of patients by establishing the precise location of the mass, and (d) defining the cystic nature of the mass and excluding other etiologies.
\end{abstract}

"Foregut cyst" is a term often used to encompass bronchogenic cyst, esophageal duplication, and enteric cyst. Enteric cysts are recognizable because they are often associated with vertebral anomalies. Bronchogenic cyst and esophageal duplication have similar radiographic appearance and also the pathologic characteristics often overlap. Foregut cysts result from abnormal budding of the foregut and constitute approximately $14 \%$ of all thoracic masses in children [1].

Their clinical presentation is varied, ranging from the asymptomatic child in whom a mass is seen in the chest radiograph to the one who presents with symptoms of airway compression $[2,3]$, or signs of compression of vascular structures such as pulmonary arteries [4] or superior vena cava.

The standard procedures for the evaluation of a child with a foregut cyst consist of a chest radiograph and a barium esophagogram. Although this radio- graphic evaluation is often sufficient, some patients experience unwarranted delays in the diagnosis and treatment. In addition others are submitted to unnecessary "work-up" because the presence of a tumor is seriously considered.

The purpose of this report is to describe the CT appearance of foregut cysts and the role of CT in the evaluation of children with foregut cyst.

\section{Material and methods}

Ten children were evaluated with CT at Children's Memorial Hospital, Chicago. The age range was 6 months to 10 years with a mean age of 5 years.

All patients had chest radiographs and six patients had barium swallow. CT was performed on the EMI5005 in seven patients and in three patients with a third generation scanner. Six patients had contrast administered during the performance of the CT scan. of the four patients without contrast administered, one had an intrapulmonary cyst, another had the CT performed in an outside institution, and the remaining two were performed early in the application of CT for evaluation of mediastinal masses.

\section{Results}

The cyst was visualized on CT in all patients. In five of the six patients who received contrast, the administration of contrast established the cystic nature of the mass. The cystic nature of the mass was established by detecting a sharply marginated low attenuation avascular mass rather than the evaluation of CT numbers. The equivocal cystic appearance in the sixth patient could be attributed to the use of the EMI 5005 instead of the third generation scanner with faster scanning times.

Besides visualizing the cyst in all patients, CT provided information which contributed to the management of six patients. 

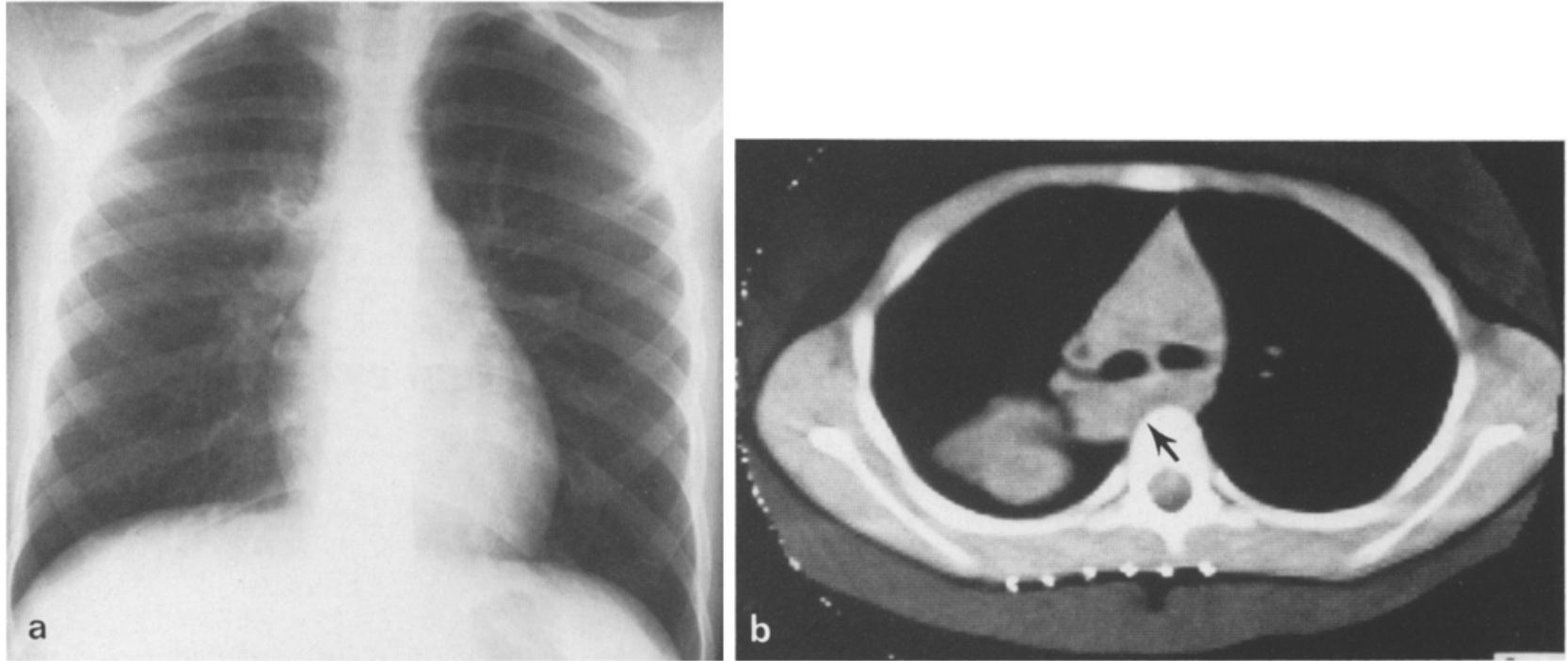

Fig. 1. a Nine-year-old child with a history of repeated episodes of right upper lobe infiltrate. Bronchoscopy and bronchography were normal. Frontal radiograph of the chest obtained between episodes demonstrates almost complete resolution of the right upper lobe infiltrate. No mediastinal masses were appreciated. b CT demonstrates a mass behind the right upper lobe bronchus (arrow). Since no intravenous contrast was injected the cystic nature could not be ascertained
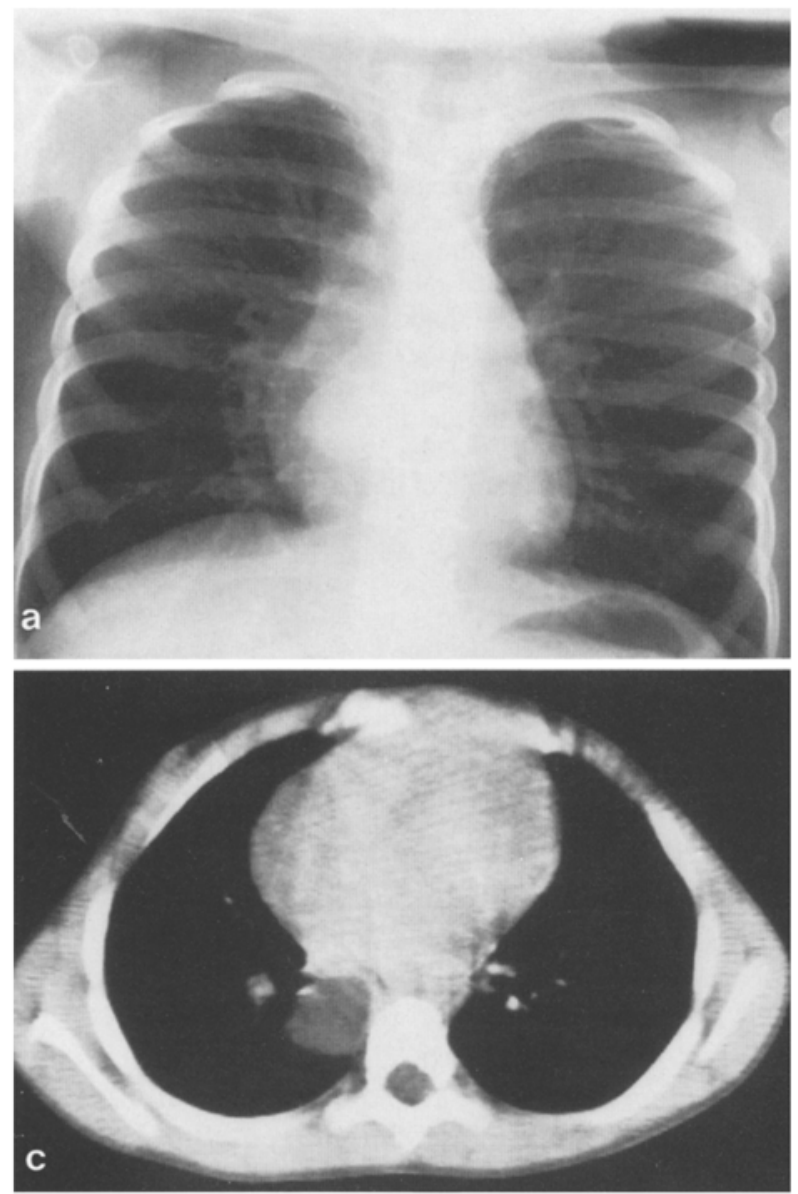

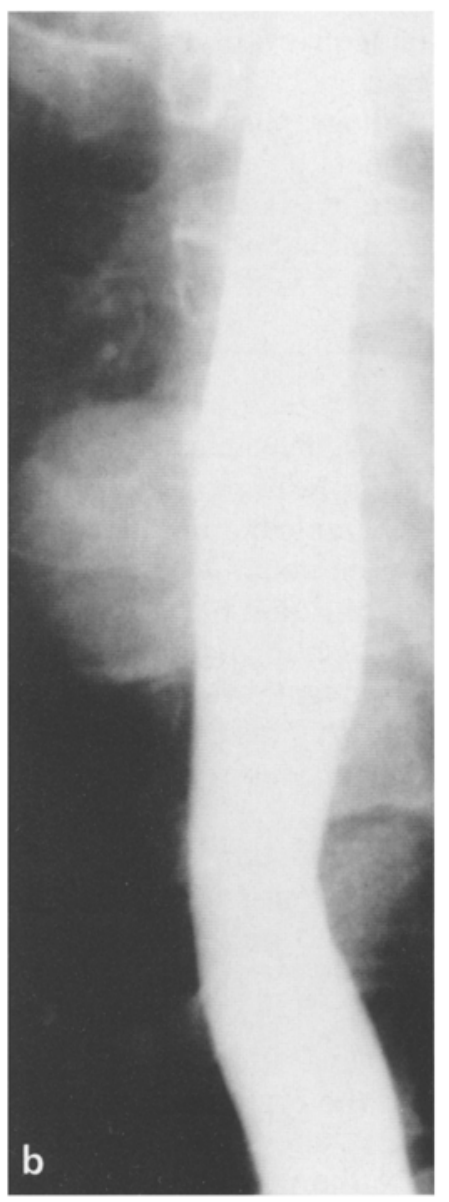

Fig. 2. a Four-year-old girl with a mass found on the frontal radiograph. b The esophagus appears to be unaffected by the esophageal duplication. c CT clearly establishes the cystic nature of the mass 

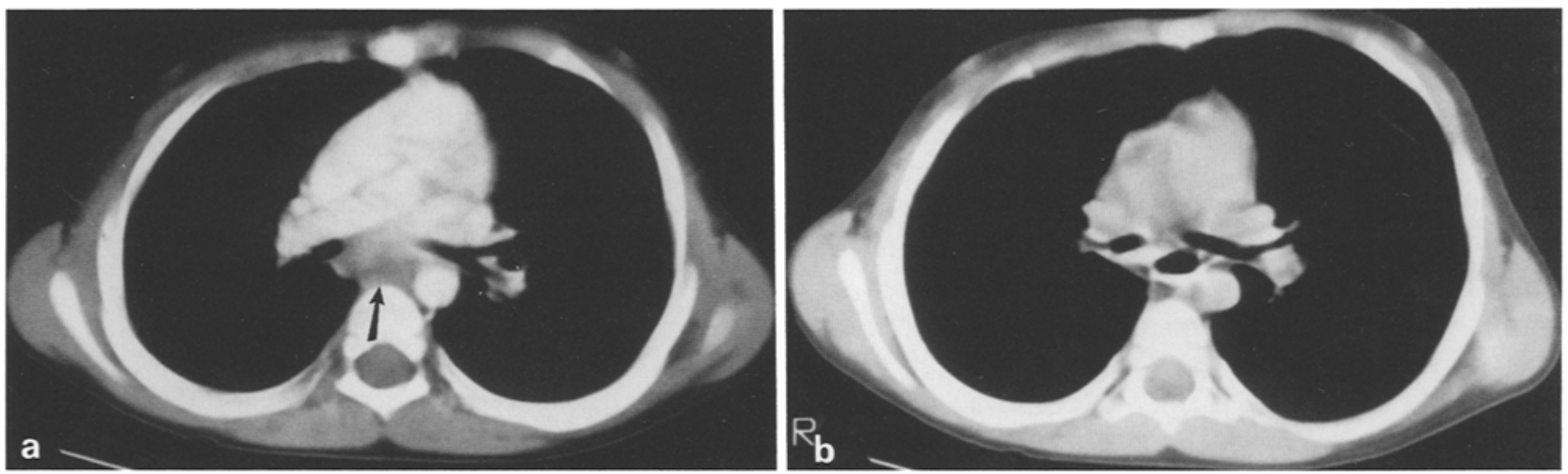

Fig. 3. a Nonenhancing mass seen in the azygoesophageal recess (arrow) after the administration of contrast. b CT at the same level after the patient is instructed to swallow contrast. The esophagus is outlined by contrast and air, and clearly was responsible for the mass

\section{The contributions of CT can be categorized:}

a) Depicting a cryptic foregut cyst. The presence of a cystic mass in a child with symptoms of airway obstruction was demonstrated despite a normal chest radiograph in two patients (Figs. $1 \mathrm{a}, \mathrm{b}$ ).

b) Clarifying abnormal radiographs in two children. The information provided by $\mathrm{CT}$ could not be ascertained, even at the time of surgery in one of the children.

c) Determining the exact location of the mass and its relation to the paravertebral gutter. CT prevented the unnecessary workup for possible tumor in two patients, despite the appearance in the chest radiograph of a posterior mediastinal mass.

d) Establishing the cystic nature of a mass in four patients, CT was able to eliminate other etiologies such as lymph nodes, dilated aorta or pulmonary arteries (Figs. $2 \mathrm{a}-\mathrm{c}$ ).

Of the ten patients, two had expeditious treatment, that is, operative excision performed at six days to three weeks after onset of symptoms. The remaining eight had delayed treatment. The CT scans were performed shortly before the surgical excision of the cysts, and were instrumental in the institution of surgical therapy.

\section{Discussion}

The presentation of a foregut cyst may vary from the asymptomatic mass found incidentally, to the mass which produces symptoms related to airway obstruction such as repeated pneumonias, obstructive emphysema, and wheezing. Occasionally they may produce compression of the superior vena cava or pulmonary arteries such as in one of our patients who had signs of peripheral pulmonary artery stenosis.
The chest radiograph and barium esophagogram are the standard radiographic procedures used in the evaluation of children with foregut cyst. The inadequacy of this approach was evident in our patients where only two of the ten patients had expeditious treatment. Similar experience has been documented in several studies $[2,5]$.

The appearance of a bronchogenic cyst on CT varies from a mass with attenuation values near those of water to values not unlike those of a solid mass [6-9]. The contents of the cyst dictate the appearance on the CT.

Bronchogenic cysts due to their cystic nature do not enhance with contrast [8], and occasionally may have calcification in the wall of the cyst. In all the patients in our series where contrast was administered, the low attenuation characteristics of the mass were evident, although in the CT scans without contrast they had a density similar to the mediastinal structures.

CT is helpful in detecting the presence of the cyst in a child with symptoms of airway obstruction, even if the conventional radiographs are normal (Fig.1). Foregut cyst may sometimes blend with the mediastinal silhouette, with little alteration of the mediastinum in the unenhanced scan. It is mandatory, when evaluating the mediastinum for a possible mediastinal cyst, that contrast be administered intravenously. In evaluating the mediastinum, a possible source of confusion is the presence of a dilated esophagus with refluxing gastric contents. This can give origin to an apparent mass similar to a duplication in the azygoesophageal recess (Fig. 3 a). To rule out this possibility contrast is administered orally, or in the older patient the simple act of swallowing may distend the esophagus with air and rule out the presence of a mass (Fig. 3b).

In those patients where the radiograph is abnormal but unclear, CT is helpful by adding a cross-sec- 
tional view. The presence of a foregut cyst cannot always be established with certainty from the frontal radiograph and the barium esophagogram. In addition, the relationship between the pathological process in the lung parenchyma and the foregut cyst can be established.

CT is useful in establishing the cystic nature of the mass and ruling out other etiologies such as lymph nodes, dilated aorta or pulmonary arteries. This information is relevant since it will guide the workup of the patients. CT, by determining the exact location of the mass, can also suggest the likelihood of a mass being neurogenic or non-neurogenic in origin. This information is important since tumor workup (VMA, myelogram, IVP, etc.) would not be performed in a patient having a low probability of having a neurogenic tumor.

$\mathrm{CT}$ is helpful in delineating the extent of the lesion [10]. Any tubular duplications with extension into the abdomen may benefit from accurate delineation of the extension so appropriate surgical approach is performed.

Ultrasound can demonstrate foregut cyst around the left atrium in the subcarinal location by imaging through the heart [11]. In one of the patients reported by Ries [11] the mass appeared solid. Lymphadenopathy can appear as a hypoechoic mass and be confused with a cystic mass [12].

In summary, the precise diagnosis of foregut cyst cannot always be made by standard radiographic means. CT is useful in the following:

a) depicting cryptic foregut cysts

b) clarifying abnormal radiographs

c) avoiding unnecessary workup of patients by establishing the precise location of the mass

d) establishing the cystic nature of the mass and excluding other etiologies, i.e., lymph nodes, dilated aorta, etc.

\section{References}

1. Bower RJ, Kiesewetter WB (1977) Mediastinal masses in infants and children. Arch Surg 112: 1003

2. Eraklis AJ, Griscom NT, McGovern JB (1969) Bronchogenic cysts of the mediastinum in infancy. New Engl $J$ Med 281: 1150

3. Alshabkhoun S, Starkey GWB, Asnes RA (1967) Bronchogenic cysts of the mediastinum in infancy. A cause of acute respiratory distress. Ann Thorac Surg 4: 532

4. Watts WJ, Rotman HH, Patten GA (1984) Pulmonary artery compression by a bronchogenic cyst simulating congenital pulmonary artery stenosis. Am J Cardiol 53:347

5. Ramenofsky ML, Leape LL, McCauley RGK (1979) Bronchogenic cyst. J Pediatr Surg 14: 219

6. Mendelson DS, Rose JS, Efremidis SC, Kirschner PA, Cohen BA (1983) Bronchogenic cysts with high CT numbers. AJR 140: 463

7. Weiss LM, Fagelman D, Warhit JM (1983) Case report. CT demonstration of an esophageal duplication cyst. J Comput Assist Tomogr 7: 716

8. Nakata H, Nakayama C, Kimoto T, Nakayama T, Tsukamato Y, Nobe T, Suzuki H (1982) Computed tomography of mediastinal bronchogenic cysts. J Comput Assist Tomogr 6:733

9. Marvasti MA, Mitchell GE, Burke WA, Meyer JA (1981) Misleading density of mediastinal cysts on computerized tomography. Ann Thorac Surg 31: 167

10. Amendola MA, Shirazi KK, Brooks J, Agha FP, Dutz W (1982) Transdiaphragmatic bronchopulmonary foregut anomaly: "dumbbell" bronchogenic cyst. AJR 138: 1165

11. Ries T, Currarino $G$, Nikaidoh H, Kennedy L (1982) Real-time ultrasonography of subcarinal bronchogenic cysts in two children. Radiology 145: 121

12. Walsh TK, Vacek JL, Bellinger RL (1985) Sarcoidosis mimicking Cor Triatriatum. Echolucency of adenopathy due to sarcoidosis. Am J Med 78: 501

Received: 23 July 1986; accepted: 1 October 1986

Dr. R.J. Hernandez

Department of Radiology

Section of Pediatric Radiology

University of Michigan Hospitals

C.S. Mott Children's Hospital

1500 E. Medical Center Drive

Ann Arbor, Michigan 48109-0252

USA

\section{Literature in pediatric radiology*}

Investigative Radiology (Philadelphia)

Pediatric radiology: Advances and current practices. Griscom, N. T. (Dept. of Rad., The Children's Hosp., 300 Longwood Ave., Boston, Massachusetts 02115, USA) 21, 833 (1986)

Journal of Bone and Joint Surgery. American Vol. (Boston)

Osteomyelitis in Gaucher disease. Bell, R.S, et al. (St. Michael's Orth. Associates, 55 Queen Street East, Suite 800, Toronto, Ontario M5C 1R5, Canada) 68-A, 1380 (1986)

Journal of Computer Assisted Tomography (New York)

MR imaging of osteoid osteoma. Glass, R. B.J. et al. (Poznanski, A.K. Dept, of Rad., The Children's Memorial Hosp., 2300 Children's Plaza, Chicago, IL 60614, USA) 10,1065 (1986)

Journal of Neurosurgery (Baltimore)

Diagnosis and management of pediatric brain-stem gliomas. Stroink, A.R.

* Compiled by Professor E. Willich, Heidelberg et al. (Hoffman, H.J., Div. of Neurosurgery, The Hosp. for Sick Children, 555 Univ. Ave., Toronto, Ontario M5G 1X8, Canada) 65, 745 (1986)

Intraspinal lipomas with spina bifida. Pierre-Kahn, A. et al. (Hồpital des Enfants Malades, 149 rue de Sèvres, F-75743 Paris Cedex 15, France) $65,756(1986)$

Dermoid tumors occuring at the site of previous myelomeningocele repair. Scott, R. M. et al. (Dept. of Neurosurgery, New England Med. Center Hosp., Inc., 171 Harrison Ave., Boston, Massachusetts 02111, USA) 65, 779 (1986)

Normal computerized tomography scans in severe head injury. Lobato, R.D. et al. (Servicio Neurocirugia, Ciudad Sanitaria " 10 de Octubre", Carretera de Andalucia, km. 5400, Madrid, Spain) 65, 784 (1986)

Temporoparietal craniopagus. Case report and review of the literature. $\mathrm{Bu}-$ cholz, R. D. et al. (Sect. of Neurological Surgery, St. Louis Univ. School of Med., 1325 South Grand Blv., St.Louis, MO 63104, USA) 66, 72 (1987) 